\title{
Development of a Genomic Resource and Identification of Nucleotide Diversity of Yellow Perch by RAD Sequencing
}

\author{
Liang Guo ${ }^{1,2}$, Hong Yao ${ }^{1}$, Brian Shepherd ${ }^{3}$, Osvaldo J. Sepulveda-Villet ${ }^{3,4}$, Dian-Chang Zhang ${ }^{2}$ \\ and Han-Ping Wang ${ }^{1 *}$ \\ ${ }^{1}$ Aquatic Genetics and Breeding Laboratory, Ohio State University South Centers, Piketon, OH, United States, ${ }^{2}$ Key \\ Laboratory of South China Sea Fishery Resources Exploitation and Utilization, Ministry of Agriculture and Rural Affairs, South \\ China Sea Fisheries Research Institutes, Chinese Academy of Fishery Sciences, Guangzhou, China, ${ }^{3}$ USDA-ARS-School of \\ Freshwater Sciences, University of Wisconsin-Milwaukee, Milwaukee, WI, United States, ${ }^{4}$ School of Freshwater Sciences, \\ University of Wisconsin-Milwaukee, Milwaukee, WI, United States
}

OPEN ACCESS

Edited by:

Nguyen Hong Nguyen, University of the Sunshine Coast,

Australia

Reviewed by:

Carolina Penaloza,

University of Edinburgh,

United Kingdom

Ricardo Perez-Enriquez,

Center for Biological Research of the Northeast (CIBNOR), Mexico

${ }^{*}$ Correspondence:

Han-Ping Wang

wang.900@osu.edu

Specialty section:

This article was submitted to

Livestock Genomics,

a section of the journal

Frontiers in Genetics

Received: 09 November 2018 Accepted: 18 September 2019

Published: 14 October 2019

Citation:

Guo L, Yao H, Shepherd B, Sepulveda-Villet OJ, Zhang D-C and Wang H-P (2019) Development of a

Genomic Resource and Identification

of Nucleotide Diversity of Yellow

Perch by RAD Sequencing.

Front. Genet. 10:992.

doi: 10.3389/fgene.2019.00992
Keywords: RAD-seq, yellow perch, polymorphic SSR, germplasm collection, genotyping, aquaculture, conservation

\section{INTRODUCTION}

Yellow perch, Perca favescens, is a freshwater fish, natively distributed in temperate and subarctic areas of North America, and its abundance and native distribution center are in the lower Great Lakes region (Craig, 1987; Sepulveda-Villet et al., 2009). Its long-term population distribution has been shaped by global climate change, mainly by Pleistocene glaciations and geophysical modifications (Sepulveda Villet and Stepien, 2012), with short-term population dynamics influenced by factors such as adaptive competition and capture fisheries (Coots, 1956; Malison, 2003; Marsden and Robillard, 2004; Houde et al., 2014; Bodamer Scarbro, 2014). During the Pleistocene glaciations, populations persisted in the three primary North American glacial refugia: Missourian, Mississippian, and Atlantic. Current yellow perch populations are attributed to at least two primary glacial refugia and divided into six major geographic regions: Northwest Lake Plains, Great Lakes watershed, Lake Champlain, US North Atlantic coastal, South Atlantic coastal, and Gulf coastal (Sepulveda Villet and Stepien, 2012). This species is in high demand for human consumption in the Great Lakes Region and a high-priority species for aquaculture production (Malison, 2003). The production of the species, however, largely depends on capture fisheries in the United States and Canada, principally the Great Lakes. While the demand for this species is approximately 5 kilotons each year, its production in aquaculture is only 100 tons each year, according the record of food and agriculture organization of the United Nations (Malison, 2003; FAO, 2018). In addition, wild harvest drastically declined from the peak harvest in the 1950s and 1960s, and even more so during the 1980s and 1990s (Malison, 2003). All these factors, including the large population fluctuation, sharp capture production decline, and high demand in aquaculture, put pressure on the basic need for genetic research, broodstock management and resource conservation.

Previous studies utilized allozymes, mitochondrial DNA, and single-sequence repeats (SSRs) as genetic markers to characterize population genetic structure (Leclerc et al., 2000; Brown et al., 2007). Restriction site-associated DNA sequencing (RAD-Seq) has emerged as a powerful technique for high-throughput single-nucleotide polymorphism (SNP) discovery and genotyping (Baird et al., 2008). For paired-end RAD reads assemblies, usually the forward reads are first clustered, whether the data are from an individual (Wang et al., 2016) or population (Hohenlohe et al., 2013), and then the reverse reads within the same cluster are assembled according to the paired-end relationships. 
SSRs have been widely used in fisheries for resource investigation and management and in aquaculture for strain identification, parentage assignment, genetic linkage map construction, and quantitative trait loci mapping (Sundaray et al., 2016). In the traditional approach of SSR development, the repeat sequences were first enriched by hybridization with biotinylated oligonucleotides and then sequenced (Chistiakov et al., 2005). With more genome and EST sequences released with the aid of Sanger sequencing and next generation sequencing (NGS) technology, SSR motifs can be searched in sequence databases (Zhan et al., 2009). However, converting these motifs to SSR markers still needs validation for polymorphism and actual polymerase chain reaction (PCR) amplification. Fortunately, SSRs still catch attention and could be directly genotyped in a sequenced population (Tang et al., 2008; Cardoso et al., 2013; Willems et al., 2014; Cantarella and Agostino, 2015; Vukosavljev et al., 2015). Furthermore, another important reason to focus on SSRs is to expand our capacity to understand SSR evolution and their influence on traits (Willems et al., 2014).

Herein, we applied RAD-Seq data for polymorphic SSR development, with the aim of showing how these sequences and markers would benefit the yellow perch conservation and aquaculture genomics research. First, we combined the advantage of longer sequence length in MiSeq platform and higher throughput of the HiSeq platform to assemble the RAD-Seq contigs. Then, a large amount of SNPs and SSRs were genotyped. Third, nucleotide diversity was assessed using developed SNPs. Fourth, a random subset of newly discovered SSRs was validated by PCR.

\section{DATA}

We applied RAD-Seq to yellow perch geographic demes to develop large numbers of polymorphic genetic markers, including SNPs and SSRs, and to evaluate nucleotide diversity of this species. We achieved 179.9 $\mathrm{M}$ read pairs in total and 6.3 $\mathrm{M}$ in average on HiSeq platform, and 2.4 $\mathrm{M}$ read pairs and 0.8 $\mathrm{M}$ in average on MiSeq platform. The average coverage was 11.2 fold in forward reads. In average, $1.0 \%$ and $37.6 \%$ reads from HiSeq and MiSeq platforms contained adaptor sequences at the $3^{\prime}$ end. In total, reads allocated into 351,578 RAD-tags were selected to assemble contigs, and 258,056 pairs of forward and reverse contigs were merged to final contigs, in which 56,845 $(22 \%)$ contained the separator of 10 "N." The length of the final contigs was $605 \pm 71 \mathrm{bp}$ (mean \pm SD, Figure 1C), and the total length of all final contigs summed was approximately 152 $\mathrm{Mbp}$, which accounts for $16.9 \%$ of the genome sequence length ( $C$ value: 0.92; Peterson et al., 1994). The lengths of the contigs assembled from both Miseq and HiSeq reads (617 $\pm 63 \mathrm{bp}$, mean \pm SD) were longer than those only from HiSeq reads $(546 \pm 96 \mathrm{bp}$, mean $\pm \mathrm{SD})(p<0.001$, two-sample Wilcoxon tests); $40.3 \%, 19.1 \%, 18.2 \%$, and $3.1 \%$ contigs mapped to the genomes of European seabass, Nile tilapia, three-spined stickleback, and zebrafish, respectively, in which the rank was consistent with the expected order of taxon.
Variants were detected, and three variant datasets were used for primer design, genetic diversity estimation, and population structure inference, respectively. The first and second datasets contained 41,736 and 33,186 SNPs, respectively. They were both uniformly located on the forward contig (Figure 1D). There were no SNPs located on enzyme recognition sites, or any inflation at end of the forward contig. The second dataset from 18 individuals was used to estimate genetic diversity, and each individual contained at least 50\% of the total SNPs (Figure 1E). The total site number was $4,442,464$, and the total nucleotide diversity was estimated as 0.00304 with $95 \%$ confidence intervals from 0.00303 to 0.00304 . The third dataset contained 27,868 SNPs and was used to inference population structure. A principal component analysis was performed, and only the first component was significant ( $p=0.027$ ), which explains $45.8 \%$ of the total variance (Figure 1F). The principal component analysis distinguished the origin and distribution of the strains examined, wherein the NY, PA, and NC1 strains were inferred to originate from the Atlantic refugium, and the NE populations were inferred to originate from the Missourian refugium. The NY strains were distributed in the North Atlantic region, while the PA and NC1 strains belonged to the South Atlantic coastal region (Table 1). The first principal component reflected the migration origin of the strains. The second principal component separated the divergence along the Atlantic coastal region. The phylogenetic tree also showed the main divergence of origin of different strains (Figure 1G).

Among the total 255,305 contigs (length <800 bp), 42,752 (16\%) contained 59,766 perfect SSRs, including 49,052 (82.1\%), $6,299(10.5 \%), 3,960(6.6 \%), 314(0.5 \%)$, and $141(0.2 \%)$ of di-, tri-, tetra-, penta-, and hexa-nucleotide repeat motifs, respectively. These repeat motifs classified into four dimeric, 10 trimeric, 30 tetrameric, 50 pentameric, and 44 hexameric categories. The most common motifs of di-, tri-, tetra-, penta-, and hexa-nucleotide repeats consisted of AC/GT (68.5\%), AAT/ ATT (38.5\%), AGAT/ATCT (23.6\%), AGAGG/CCTCT (19.4\%), and ACACGC/CGTGTG (36.9\%) motifs. When considering imperfect SSRs, then the total number of SSRs increased to 73,703, and the most common repeats were changed to be AC/ GT, AAT/ATT, AAAT/ATTT, AATTC/AATTG, and AACCCT/ AGGGTT motifs. We took allele number as a measurement to evaluate the polymorphism of each type of motif. A total of 10,412 SSRs were then detected with at least two alleles. As with other studies in humans (Willems et al., 2014), the number of alleles is inversely correlated with motif length $(p<0.001$, Kruskal-Wallis test) and positively correlated with length of alleles (Pearson correlation coefficient: 0.23, $p<0.001$ ). To explore the genomic resource in yellow perch, primers were successfully designed for 3,830 SSRs with at least three alleles and flanked with sequence at least $200 \mathrm{bp}$ at each side. The randomly selected 40 pairs of primers were validated using PCR, and $34(85 \%)$ pairs showed expected bands, in which three pairs showed extra tidy bands outside the expected range (Figure 1H). The high validation ratio showed the assembled contigs were reliable, and the designed primers could be directly used in genotyping. 


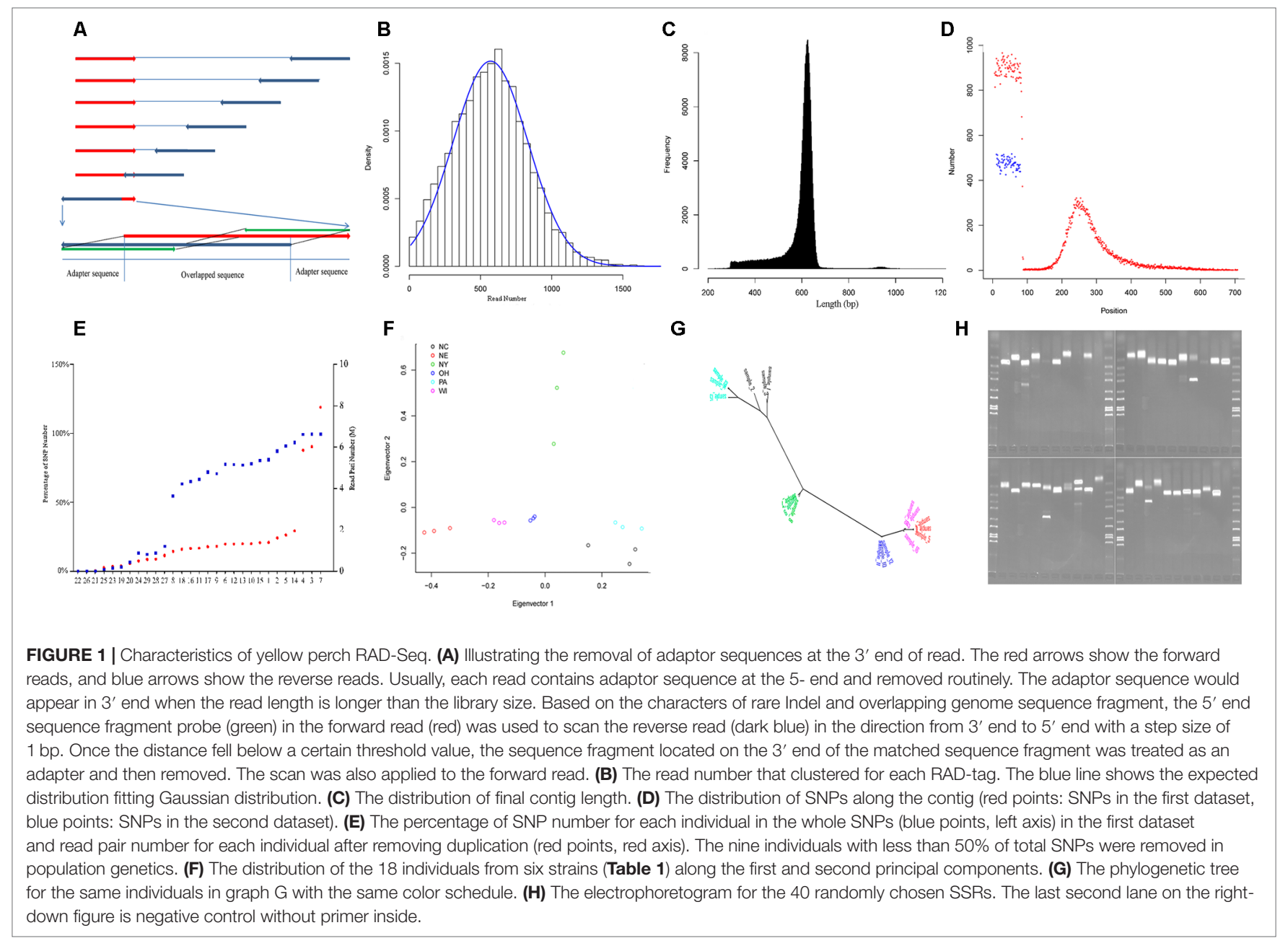

\section{MATERIALS AND METHODS}

\section{Sample Collection}

Eight strains, NC1 (Perquimans River, 2010), NC2 (Perquimans River, 2006), NE (Sandhill lakes), NY (Erie Canal), MD (Choptank River), OH (Lake Erie), PA, and WI (Green Bay), were selected with three to four individuals sampled from each strain (Table 1). These samples captured the mainly native distribution region of this species. Genomic DNA was extracted from fin tissues using the method described by Li et al. (2007). All the samples were sequenced using paired-end RAD-Seq (Baird et al., 2008), in which three individuals were sequenced on MiSeq platform with $2 \times 300 \mathrm{bp}$ and others on HiSeq 2000 platform with $2 \times 100 \mathrm{bp}$. The restriction enzyme was EcoRI, and the library size was approximately $600 \mathrm{bp}$.

\section{Contig Assembly}

Reads were filtered and clustered using software Stacks version 1.42 (Catchen et al., 2013). The raw reads were filtered and separated using program process_radtags with default parameters without rescue of barcodes. Then, the forward reads were cut to be $85 \mathrm{bp}$ in length. The reads from each individual were clustered using programs denovo_map.pl, rxstacks, cstacks, and sstacks with parameters of minimal depth for each stack, maximal mismatch allowed between stacks, and number of mismatches allowed between sample loci to be 3, 2, and 3. The highly repetitive catalogued loci were removed.

The forward and reverse reads that allocated into each RAD-tag were assembled separately, and the forward contig and reverse contig were merged into final contig. Before being allocated, the read pairs, without cutting in length, were processed using Hamming distance to filter sequence fragments that were actually adapter sequences in the $3^{\prime}$ end (Figure 1A). This step in trimming the adapter sequence was performed in the Perl script, trim_adaptorseq.pl, with sizes of probe 50bp and maximal distance threshold 5. Then the reads belonging to each RAD-tag (Figure 1B) were allocated using sort_read_pairs. $\mathrm{pl}$ and assembled separately with software CAP3 (Huang and Madan, 1999) with the following default parameters: $(d=500$, $g=2, \mathrm{~h}=100,000, I=30, j=31, n=-2, s=800, t=3000$, $o=16, p=80, r=0, y=50, z=5)$. The forward and reverse contigs that were supported by at least $10 \%$ and $60 \%$ reads, respectively, were merged using the Needleman-Wunsch global alignment algorithm (Needleman and Wunsch, 1970), in which 
TABLE 1 | Description of samples and statistic of reads.

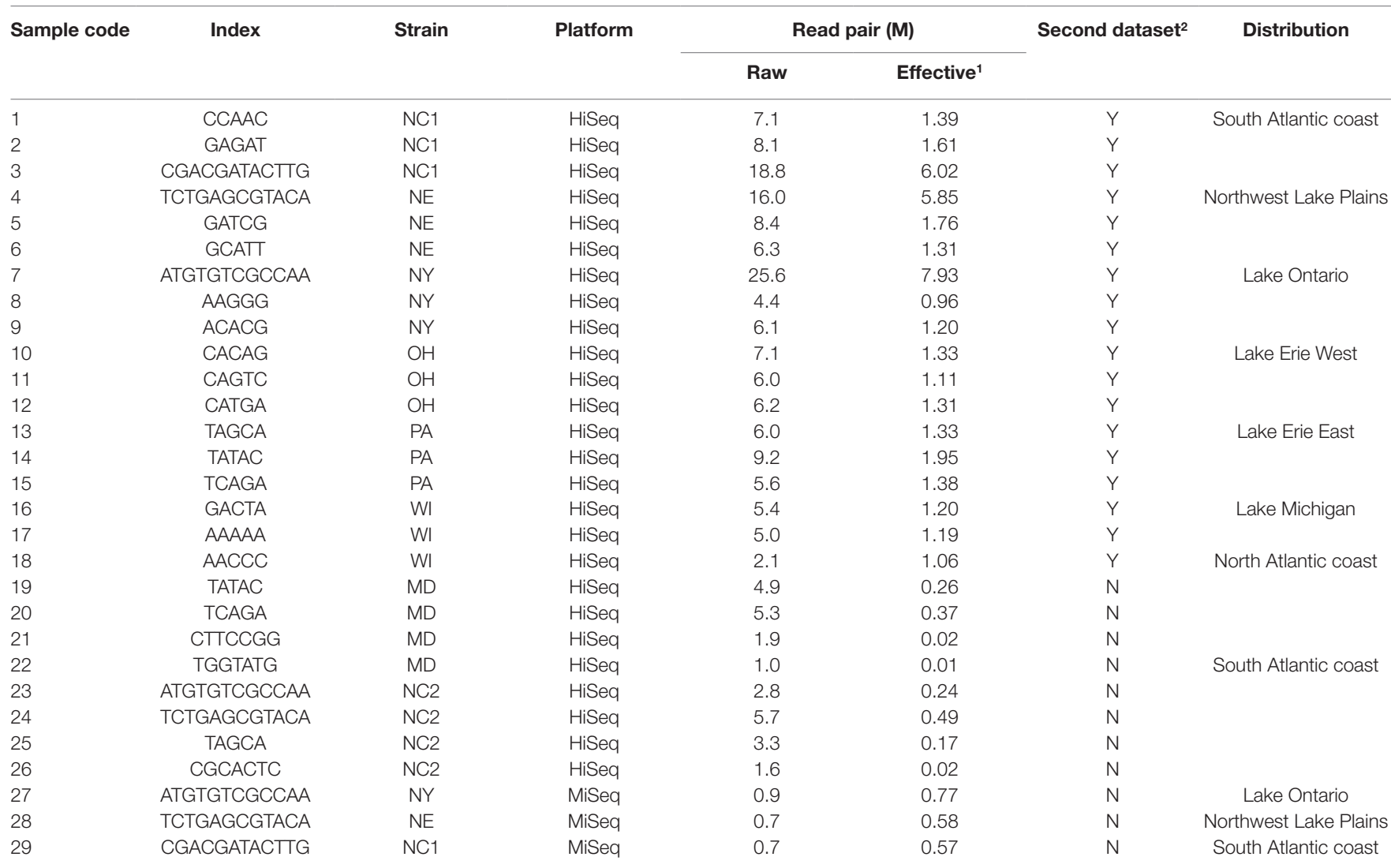

${ }^{1}$ This column shows the number of read pairs after removing of duplication.

${ }^{2} Y$ indicates the individual was included in the second dataset, otherwise represented by " $N$ ".

the exact match achieves a score of 5 . The merged contigs were considered as overlapped with the following conditions: the 10 headmost bases identical to the 10 headmost base in forward contig; the 10 backmost bases identical to the 10 backmost bases in reverse contig; the score larger than 50; the identity larger than 10; and the quotient of the score divided by the identity large than 4 . The nonoverlapped forward contig and reverse contig were connected with 10 "N" as a separator.

The final contigs were investigated by comparing them with other reference genomes and counting SSR motifs. Four related fish genomes were selected for this purpose: three-spined stickleback (ASM18067v1) (Berner et al., 2019), Nile tilapia (Orenil1.1) (Brawand et al., 2014), European seabass (seabass_ V1.0) (Tine et al., 2014), and zebrafish (GRCz10) (Howe et al., 2013). European seabass and yellow perch belong to the same suborder of Percoidei, and Nile tilapia and yellow perch belong to the same order of Perciformes, while three-spined stickleback and zebrafish belong to order of Gasterosteiformes and Cypriniformes, respectively. Blastn (Altschul et al., 1997) was used to align the sequences with $e$ value $10^{-6}$ and of at least $100-$ bp alignment length.

\section{SNP and Indel Identification}

The final contigs were connected with 100 "N" to be a pseudomolecule as the reference sequence. The original reads without trimming lengths were mapped to the reference sequence with the BWA-MEM software package version 0.7.15 (Li, 2013) with a limitation of a maximum insert size of $1 \mathrm{kbp}$, which was also used for polymorphic SSR detection below. SAMtools version 1.3.1 (Li et al., 2009) and Picard tools version 2.3.0 (http://picard.sourceforge.net) were used to manipulate the mapped files. Prior to calling markers, reads with an insert size over $1,000 \mathrm{bp}$ were removed using an in-house Perl script, and properly paired reads were then selected using SAMtools (Li et al., 2009). Duplicated reads including optical duplications were filtered using Picard tools. Reads with Indels were realigned with RealignerTargetCreator and IndelRealigner of the GATK software package version 3.6 (McKenna et al., 2010). SNPs and Indels were called using SAMtools ( $\mathrm{Li}$ et al., 2009) in a multiple-sample model and filtered using VCFtools version 0.1.15 (Danecek et al., 2011) with the following parameters: (1) base quality and map quality $\geq 20$; (2) variant quality $\geq 300$; (3) depth per sample $\geq 5$-fold and <200-fold; (4) minor allele frequency $>0.05$. Three variant datasets were obtained with different and further filter. In the first dataset, the SNPs and Indels that existed in at least 10 individuals were selected. In the second dataset, 11 individuals were removed because of containing too few SNPs (Figure 1E). The remaining individuals were from six strains, each with three individuals (Table 1), and 
they could still represent the native population of this species. Those SNPs that existed in at least $80 \%$ of individuals and located from 11 to $80 \mathrm{bp}$ on the forward contig were selected. In the third dataset, only one SNP from each forward contig in second dataset was selected.

\section{Population Genetics}

The second dataset was used to estimate nucleotide diversity, including per-SNP nucleotide diversity $\left(\pi_{\mathrm{SNP}}\right)$ and total nucleotide diversity $\left(\pi_{\text {total }}\right)$. Per-SNP nucleotide diversity was calculated using VCFtools version 0.1.15 (Danecek et al., 2011), and total nucleotide diversity was averaged across all the sites, including the invariant sites that meet the minimum depth in each individual and minimum percentage in all the 18 individuals (Lozier, 2014). Confidence interval for total nucleotide diversity was obtained by 10,000 bootstrap replicates across sites using package boot (Canty and Ripley, 2012) in R (Team, 2013).

The third dataset was used to infer the population structure. A phylogenetic tree by the maximum likelihood method was constructed using SNPhylo version 20140701 (Lee et al., 2014), and a principal component analysis was performed in R program LEA (Frichot and François, 2015), in which significance of the identified principal components was evaluated through TracyWidom statistics.

\section{Polymorphic SSRs Detection and Validation}

Two programs were used to search for SSRs in the final contigs, Microsatellite search module (MISA, http://pgrc.ipkgatersleben.de/misa/) and Tandem Repeats Finder version 4.07b (TRF) (Benson, 1999). MISA takes usage of regular expression pattern to scan the contigs from perfect SSRs, including di-, tri-, tetra-, penta-, and hexa-nucleotide motifs with numbers of uninterrupted repeat units more than 5, 4, 4,4 , and 4, respectively. TRF took usage of alignment score to recognize SSR with the following parameters: a match weight $=2$; a mismatch and Indel penalty $=7$; probability of a matching $=80 \%$; probability of an Indel $=10 \%$; maximum period $=500$; the minimum scores for di-, tri-, tetra-, penta-, and hexa-nucleotide motifs $=22,28,28,32$, and 34, respectively (Benson, 1999; Willems et al., 2014).

Polymorphic SSRs were detected using the software lobSTR version 3.0.3 (Gymrek et al., 2012) according to the best practice. The SSR motifs were defined based on the result of TRF (Benson, 1999; Willems et al., 2014). The lobSTR genotyped SSRs with the following options: min-het-freq $=0.2$, min-border $=5$, $\min$ bp-before-indel $=7$, maximal-end-match $=15$, min-read-endmatch $=10$, and max-matedist $=1000$.

The raw genotyped SSRs with quality of at least 300, at least three alleles, and at least $200 \mathrm{bp}$ flanking sequence in each side were selected as high-quality SSRs. Primers were designed using Primer 3 (Andreas et al., 2012) in batch with SNPs and Indels being masked (the first dataset). The parameters were set as follows: (1) primer length ranging from 18 to 24 bases with optimal sizes of $21 \mathrm{nt}$; (2) PCR product size ranging from 125 to $250 \mathrm{bp}$; (3) melting temperature between $55^{\circ} \mathrm{C}$ and $65^{\circ} \mathrm{C}$, with $60^{\circ} \mathrm{C}$ as the optimum annealing temperature; (4) a GC content of $40 \%$ to $60 \%$, with an optimum of $50 \%$. The premier pairs were in silico validated using re-PCR (Schuler, 1998) with parameters of two mismatches and two gaps, and those with only one production that existed were treated as high-quality primers. Forty pairs of primers were selected at random and then synthesized in Integrated DNA Technologies (Coralville, IA). The PCR reaction was conducted using Platinum ${ }^{\mathrm{TM}}$ SuperFi ${ }^{\mathrm{TM}}$ Green PCR Master Mix (Invitrogen, Carlsbad, CA) and performed in a thermal cycler (Bio-Rad, Hercules, CA) under the following conditions: $30 \mathrm{~s}$ at $98^{\circ} \mathrm{C} ; 35$ cycles of 10 $\mathrm{s}$ at $98^{\circ} \mathrm{C}, 30 \mathrm{~s}$ at $55^{\circ} \mathrm{C}, 45 \mathrm{~s}$ at $72^{\circ} \mathrm{C}$; and $5 \mathrm{~min}$ at $72^{\circ} \mathrm{C}$. PCR products were visualized in a $2 \%$ agarose gel.

\section{ETHICS STATEMENT}

All the methods and experimental protocols of this study were performed in accordance with guidelines and regulations approved by the animal ethics committee of The Ohio State University (USA) and the University of Wisconsin-Milwaukee (USA) Institutional Animal Care and Use Committee.

\section{AUTHOR CONTRIBUTIONS}

H-PW and BS conceived and designed the experiments. HY and OS-V performed the experiment. LG and D-CZ analyzed the data and prepared a draft of the manuscript. H-PW and BS revised and finalized the manuscript. All authors read and approved the manuscript.

\section{FUNDING}

This work was financially supported by United States Department of Agriculture (No. 2010-38879-20946) and USDA-ARS CRIS project (5090-31320-003-00D). Salaries were provided by state and federal funds appropriated to The Ohio State University, Ohio Agricultural Research and Development Center.

\section{ACKNOWLEDGMENTS}

The authors gratefully acknowledge the technical contribution of Dr. Yniv Palti and Dr. Guangtu Gao for generating and sequencing one of the yellow perch RAD libraries. We would also like to thank Dean Rapp and Paul O'Bryant for collecting and maintaining experimental fish throughout the experiment and Bradford Sherman for his comments on the manuscript. 


\section{REFERENCES}

Altschul, S. F., Madden, T. L., Schaffer, A. A., Zhang, J., Zhang, Z., Miller, W., et al. (1997). Gapped BLAST and PSI-BLAST: a new generation of protein database search programs. Nucleic Acids Res. 25, 3389-3402. doi: 10.1093/ nar/25.17.3389

Andreas, U., Ioana, C., Triinu, K., Jian, Y., Faircloth, B. C., Maido, R., et al. (2012). Primer3-new capabilities and interfaces. Nucleic Acids Res. 40, e115. doi: 10.1093/nar/gks596

Baird, N. A., Etter, P. D., Atwood, T. S., Currey, M. C., Shiver, A. L., Lewis, Z. A., et al. (2008). Rapid SNP discovery and genetic mapping using sequenced RAD markers. PLoS One 3, e3376. doi: 10.1371/journal.pone.0003376

Benson, G. (1999). Tandem repeats finder: a program to analyze DNA sequences. Nucleic Acids Res. 27, 573. doi: 10.1093/nar/27.2.573

Berner, D., Roesti, M., Bilobram, S., Chan, S. K., Kirk, H., Pandoh, P., et al. (2019). De novo sequencing, assembly, and annotation of four threespine stickleback genomes based on microfluidic partitioned DNA libraries. Genes 10, 426. doi: 10.3390/genes10060426

Bodamer Scarbro, B. L. (2014). The physiological and behavioral responses of yellow perch to hypoxia, University of Toledo.

Brawand, D., Wagner, C. E., Li, Y. I., Malinsky, M., Keller, I., Fan, S., et al. (2014). The genomic substrate for adaptive radiation in African cichlid fish. Nature 513, 375-381. doi: 10.1038/nature13726

Brown, B., Wang, H., Li, L., Givens, C., and Wallat, G. (2007). Yellow perch strain evaluation I: genetic variance of six broodstock populations. Aquaculture 271, 142-151. doi: 10.1016/j.aquaculture.2007.06.022

Cantarella, C., and Agostino, D. (2015). PSR: polymorphic SSR retrieval. BMC Res. Notes 8, 1. doi: 10.1186/s13104-015-1474-4

Canty, A., and Ripley, B. (2012). boot: Bootstrap R (S-Plus) functions. R package version 1.

Cardoso, S. D., Goncalves, D., Robalo, J. I., Almada, V. C., Canario, A. V., and Oliveira, R. F. (2013). Efficient isolation of polymorphic microsatellites from high-throughput sequence data based on number of repeats. Mar Genomics 11, 11-16. doi: 10.1016/j.margen.2013.04.002

Catchen, J., Hohenlohe, P. A., Bassham, S., Amores, A., and Cresko, W. A. (2013). Stacks: an analysis tool set for population genomics. Mol. Ecol. 22, 3124-3140. doi: $10.1111 /$ mec.12354

Chistiakov, D. A., Hellemans, B., Haley, C. S., Law, A. S., Tsigenopoulos, C. S., Kotoulas, G., et al. (2005). A microsatellite linkage map of the European sea bass Dicentrarchus labrax L. Genetics 170, 1821-1826. doi: 10.1534/ genetics.104.039719

Coots, M. (1956). The yellow perch, Perca flavescens (Mitchill), in the Klamath River. Calif. Fish Game 42, 219-228.

Craig, J. (1987). Biology of perch and related fish. Croom Helm; Timber Press.

Danecek, P., Auton, A., Abecasis, G., Albers, C. A., Banks, E., DePristo, M. A., et al. (2011). The variant call format and VCFtools. Bioinformatics 27, 2156-2158. doi: 10.1093/bioinformatics/btr330

FAO (2018). Cultured Aquatic Species Information Programme, American yellow perch. Available online: http://www.fao.org (accessed on 20 September 2016).

Frichot, E., and François, O. (2015). LEA: an R package for landscape and ecological association studies. Methods Ecol. Evol. 6, 925-929. doi: 10.1111/2041-210X.12382

Gymrek, M., Golan, D., Rosset, S., and Erlich, Y. (2012). lobSTR: a short tandem repeat profiler for personal genomes. Genome Res. 22, 1154-1162. doi: 10.1101/ gr.135780.111

Hohenlohe, P. A., Day, M. D., Amish, S. J., Miller, M. R., Kamps-Hughes, N., Boyer, M. C., et al. (2013). Genomic patterns of introgression in rainbow and westslope cutthroat trout illuminated by overlapping paired-end RAD sequencing. Mol. Ecol. 22, 3002-3013. doi: 10.1111/mec.12239

Houde, M., Giraudo, M., Douville, M., Bougas, B., Couture, P., De Silva, A. O., et al. (2014). A multi-level biological approach to evaluate impacts of a major municipal effluent in wild St. Lawrence River yellow perch (Perca flavescens). Sci. Total Environ. 497, 307-318. doi: 10.1016/j. scitotenv.2014.07.059
Howe, K., Clark, M. D., Torroja, C. F., Torrance, J., Berthelot, C., Muffato, M., et al. (2013). The zebrafish reference genome sequence and its relationship to the human genome. Nature 496, 498-503. doi: 10.1038/nature12111

Huang, X., and Madan, A. (1999). CAP3: a DNA sequence assembly program. Genome Res. 9, 868-877. doi: 10.1101/gr.9.9.868

Leclerc, D., Wirth, T., and Bernatchez, L. (2000). Isolation and characterization of microsatellite loci in the yellow perch (Perca flavescens), and crossspecies amplification within the family Percidae. Mol. Ecol. 9, 995-997. doi: 10.1046/j.1365-294x.2000.00939-3.x

Lee, T., Guo, H., Wang, X., Kim, C., and Paterson, A. H. (2014). SNPhylo: a pipeline to construct a phylogenetic tree from huge SNP data. BMC Genomics 15, 162. doi: 10.1186/1471-2164-15-162

$\mathrm{Li}, \mathrm{H}$. (2013). Aligning sequence reads, clone sequences and assembly contigs with BWA-MEM. arXiv:1303.3997.

Li, H., Handsaker, B., Wysoker, A., Fennell, T., Ruan, J., Homer, N., et al. (2009). The sequence alignment/map format and SAMtools. Bioinformatics 25, 20782079. doi: 10.1093/bioinformatics/btp352

Lozier, J. D. (2014). Revisiting comparisons of genetic diversity in stable and declining species: assessing genome-wide polymorphism in North American bumble bees using RAD sequencing. Mol. Ecol. 23, 788-801. doi: 10.1111/ mec. 12636

Malison, J. A. (2003). A white paper on the status and needs of yellow perch aquaculture in the north central region. North Centr. Reg. Aquacult. Center.

Marsden, J. E., and Robillard, S. R. (2004). Decline of yellow perch in southwestern Lake Michigan, 1987-1997. N. Am. J. Fish. Manage. 24, 952- 966. doi: 10.1577/ M02-195.1

McKenna, A., Hanna, M., Banks, E., Sivachenko, A., Cibulskis, K., Kernytsky, A., et al. (2010). The genome analysis toolkit: a MapReduce framework for analyzing next-generation DNA sequencing data. Genome Res. 20, 1297-1303. doi: $10.1101 /$ gr.107524.110

Needleman, S. B., and Wunsch, C. D. (1970). A general method applicable to the search for similarities in the amino acid sequence of two proteins. J. Mol. Biol. 48, 443-453. doi: 10.1016/0022-2836(70)90057-4

Peterson, D. G., Stack, S. M., Healy, J. L., Donohoe, B. S., and Anderson, L. K. (1994). The relationship between synaptonemal complex length and genome size in four vertebrate classes (Osteichthyes, Reptilia, Aves, Mammalia). Chromosome Res. 2, 153-162. doi: 10.1007/BF01553494

Schuler, G. D. (1998). Electronic PCR: bridging the gap between genome mapping and genome sequencing. Trends Biotechnol. 16, 456-459. doi: 10.1016/ S0167-7799(98)01232-3

Sepulveda Villet, O. J., and Stepien, C. A. (2012). Waterscape genetics of the yellow perch (Perca flavescens): patterns across large connected ecosystems and isolated relict populations. Mol. Ecol. 21, 5795-5826. doi: 10.1111/ mec. 12044

Sepulveda-Villet, O. J., Ford, A. M., Williams, J. D., and Stepien, C. A. (2009). Population genetic diversity and phylogeographic divergence patterns of the yellow perch (Perca flavescens). J. Great Lakes Res. 35, 107-119. doi: 10.1016/j. jglr.2008.11.009

Sundaray, J. K., Rasal, K. D., Chakrapani, V., Swain, P., Kumar, D., Ninawe, A. S., et al. (2016). Simple sequence repeats (SSRs) markers in fish genomic research and their acceleration via next-generation sequencing and computational approaches. Aquacult. Int. 24, 1089-1102. doi: 10.1007/ s10499-016-9973-4

Tang, J., Baldwin, S. J., Jacobs, J. M., van der Linden, C. G., Voorrips, R. E., Leunissen, J. A., et al. (2008). Large-scale identification of polymorphic microsatellites using an in silico approach. BMC Bioinformatics 9, 1. doi: 10.1186/1471-2105-9-374

Team, R. C. (2013). R: a language and environment for statistical computing.

Tine, M., Kuhl, H., Gagnaire, P., Louro, B., Desmarais, E., Martins, R. S. T., et al. (2014). European sea bass genome and its variation provide insights into adaptation to euryhalinity and speciation. Nat. Commun. 5, 5770. doi: 10.1038/ncomms6770

Vukosavljev, M., Esselink, G. D., van T, W. W., Cox, P., Visser, R. G., Arens, P., et al. (2015). Efficient development of highly polymorphic microsatellite markers based on polymorphic repeats in transcriptome sequences of multiple individuals. Mol. Ecol. Resour. 15, 17-27. doi: 10.1111/1755-0998.12289 
Wang, J., Xue, D. X., Zhang, B. D., Li, Y. L., Liu, B. J., and Liu, J. X. (2016). Genomewide SNP discovery, genotyping and their preliminary applications for population genetic inference in spotted sea bass (Lateolabrax maculatus). PLoS One 11, e0157809. doi: 10.1371/journal.pone.0157809

Willems, T., Gymrek, M., Highnam, G., Mittelman, D., Erlich, Y., and Consortium, G. P. (2014). The landscape of human STR variation. Genome Res. 24, 1894-1904. doi: 10.1101/gr.177774.114

Zhan, A., Wang, Y., Brown, B., and Wang, H. P. (2009). Isolation and characterization of novel microsatellite markers for yellow perch (Perca flavescens). Int. J. Mol. Sci. 10, 18-27. doi: 10.3390/ijms10010018
Conflict of Interest: The authors declare that the research was conducted in the absence of any commercial or financial relationships that could be construed as a potential conflict of interest.

Copyright (C) 2019 Guo, Yao, Shepherd, Sepulveda-Villet, Zhang and Wang. This is an openaccess article distributed under the terms of the Creative Commons Attribution License (CC BY). The use, distribution or reproduction in other forums is permitted, provided the original author(s) and the copyright owner(s) are credited and that the original publication in this journal is cited, in accordance with accepted academic practice. No use, distribution or reproduction is permitted which does not comply with these terms. 